\title{
Intra-Articular Injection of Mitomycin C Prevents Progression of Immobilization-Induced Arthrogenic Contracture in the Remobilized Rat Knee
}

\author{
Akinori KANEGUCHI ${ }^{1}$, Junya OZAWA ${ }^{1}$, Kaoru YAMAOKA ${ }^{1}$ \\ ${ }^{1}$ Department of Rehabilitation, Faculty of Rehabilitation, Hiroshima International University, \\ Higashi-Hiroshima, Hiroshima, Japan
}

Received February 21, 2019

Accepted October 17, 2019

Epub Ahead of Print December 19, 2019

\section{Summary}

This study tested whether cell cycle inhibitor mitomycin C (MMC) prevents arthrogenic contracture progression during remobilization by inhibiting fibroblast proliferation and fibrosis in the joint capsule. Rat knees were immobilized in a flexed position to generate flexion contracture. After three weeks, the fixation device was removed and rat knees were allowed to freely move for one week. Immediately after and three days after fixator removal, rats received intra-articular injections of MMC or saline. The passive extension range of motion (ROM) was measured before and after myotomy of the knee flexors to distinguish myogenic and arthrogenic contractures. In addition, both cellularity and fibrosis in the posterior joint capsule were assessed histologically. Joint immobilization significantly decreased ROMs both before and after myotomy compared with untreated controls. In saline-injected knees, remobilization increased ROM before myotomy, but further decreased that after myotomy compared with that of knees immediately after three weeks of immobilization. Histological analysis revealed that hypercellularity, mainly due to fibroblast proliferation, and fibrosis characterized by increases in collagen density and joint capsule thickness occurred after remobilization in saline-injected knees. Conversely, MMC injections were able to prevent the remobilization-enhanced reduction of ROM after myotomy by inhibiting both hypercellularity and joint capsule fibrosis. Our results suggest that joint capsule fibrosis accompanied by fibroblast proliferation is a potential cause of arthrogenic contracture progression during remobilization, and that inhibiting fibroblast proliferation may constitute an effective remedy.
\end{abstract}

\section{Key words}

Joint contracture • Immobilization • Fibroblast • Fibrosis • Mitomycin C

\section{Corresponding author}

J. Ozawa, Department of Rehabilitation, Faculty of Rehabilitation, Hiroshima International University, Kurose-Gakuendai 555-36, Higashi-Hiroshima, Hiroshima, 739-2695, Japan. Fax: +81-82370-4542. E-mail: j-ozawa@hirokoku-u.ac.jp

\section{Introduction}

Joint immobilization is frequently used to treat orthopedic disorders such as bone fractures and ligamentous injuries, but often causes joint contracture (Chesworth and Vandervoort 1995, Moseley et al. 2005, Nightingale et al. 2007), which is characterized by a reduced passive range of motion (ROM) of the joint (Wong et al. 2015). As joint contractures can lead to various types of locomotive disabilities (Bot et al. 2012, De Smet 2007), management of this impairment is a critical issue in the field of orthopedics.

Because joint immobilization is a major cause of joint contracture, it is clinically accepted that joint movement during remobilization is effective in improving joint contracture. Passive joint movements such as stretching are frequently applied in this context. In animal studies, however, it remains controversial whether stretching has beneficial effects on immobilization-induced joint contracture (Kondo et al. 2012, Okita et al. 2001, Usuba et al. 2007). In humans, 
a clinical study failed to show related benefits of passive stretching (Moseley et al. 2005), and recent reviews supported by high-quality evidence suggest that stretching does not have a clinically important role in joint contracture treatment (Harvey et al. 2017a, Harvey et al. 2017b). More reasonable and effective treatment approaches are therefore needed.

Formation and recovery processes of immobilization-induced joint contracture have been closely examined using animal models. The responsible structures have been broadly divided into myogenic and arthrogenic factors (Nagai et al. 2014, Trudel and Uhthoff 2000, Trudel et al. 2014). Trudel et al. demonstrated that a myogenic factor is mainly responsible for joint contracture in the early phases of immobilization (within two weeks) and is resolved by remobilization (Trudel et al. 2014). In contrast, arthrogenic factors largely contribute to severe joint contracture induced by prolonged (over four weeks) immobilization, and recovery by remobilization is not expected in these cases (Trudel et al. 2014). Arthrogenic contracture also further progresses during remobilization following short-term (within three weeks) immobilization (Kaneguchi and Ozawa 2017, Kaneguchi et al. 2017, Kaneguchi et al. 2018a, Kaneguchi et al. 2018b, Kaneguchi et al. 2019, Trudel et al. 2014). To avoid irreversible joint contracture, arthrogenic contracture should therefore be targeted.

Fibrosis in the joint components is believed to be the major factor contributing to development of arthrogenic contracture in injured (Fukui et al. 2000, Fukui et al. 2001, Gao et al. 2017, Li et al. 2013a) and immobilized joints (Sasabe et al. 2017). For example, administration of decorin, which suppresses bioactivity of cell adhesion, as well as fibrotic regulators, such as transforming growth factor-beta, can improve restricted joint motion in the rabbit intra-articular adhesion model (Fukui et al. 2001). However, several studies showed development of arthrogenic contracture without fibrosis in the periarticular connective tissue in spinal cord injury and immobilization models (Hagiwara et al. 2010, Kaneguchi et al. 2017, Kaneguchi et al. 2018a, Kaneguchi et al. 2018b, Kaneguchi et al. 2019, Moriyama et al. 2007). While it therefore remains controversial whether joint fibrosis is a major factor in arthrogenic contracture, development of fibrosis is observed in the joint capsule of remobilized joints together with progression of arthrogenic contracture (Kaneguchi et al. 2017, Kaneguchi et al. 2018a,
Kaneguchi et al. 2018b, Kaneguchi et al. 2019). We previously showed that anti-inflammatory treatment using the steroidal drug dexamethasone can prevent remobilization-induced arthrogenic contracture progression by suppressing joint capsule fibrosis and fibroblast proliferation (Kaneguchi et al. 2018b). Other studies using intra-articular adhesion models also reported that administration of anti-inflammatory agents such as celecoxib and botulinum toxin type A attenuates joint contracture and intra-articular adhesion as well as fibroblast proliferation (Baranowski et al. 2019, Gao et al. 2017, Li et al. 2013a). Fibroblasts produce extracellular matrix proteins such as collagens, and thus fibroblast proliferation is an important process in the development of joint fibrosis (Emami et al. 2012, Li et al. 2014). We therefore suggest that proliferation of fibroblasts triggered by inflammation plays an important role in forming joint capsule fibrosis and that inhibition of fibrosis may be essential for blocking the progression of arthrogenic contracture.

To inhibit fibroblast proliferation, we focused on the cell cycle inhibitor mitomycin C (MMC). In a clinical context, MMC is generally used as an anti-cancer drug (Kahmann et al. 2010). However, it also finds application pre-clinically and clinically as an anti-fibrotic drug for keloid, capsular contracture, and scarring after ocular surgery (Lane et al. 2003, Li et al. 2013b, Nava et al. 2017, Sidle and Kim 2011, Simman et al. 2003, Wang et al. 2012). MMC can attenuate the development of fibrosis as well as inhibit fibroblast proliferation in injury-induced intra-articular adhesion models (Kocaoglu et al. 2011, Li et al. 2013b, Wang et al. 2012).

Therefore, we hypothesized that remobilizationinduced joint capsule fibrosis and progression of arthrogenic contracture would also be attenuated by MMC administration via inhibition of fibroblast proliferation. To test this hypothesis, we investigated the effects of MMC on joint ROM and histopathology in the remobilized rat knee.

\section{Materials and Methods}

\section{Experimental animals}

Twenty-five eight-week-old male Wistar rats (190-220 g; Japan SLC, Shizuoka, Japan) were used in this study. The rats were randomly divided into four groups: control $(n=4)$, immobilization (IM: $n=7)$, remobilization with saline injections after immobilization (RM: $n=7$ ), and remobilization with MMC injections 
after immobilization ( $\mathrm{RM}+\mathrm{M}$ : $\mathrm{n}=7)$. In the control group, data from the right and left knees were treated as individual samples; therefore, we used data of eight knees from four rats as controls. Rats were housed in standard cages in a temperature-controlled room $\left(20-25^{\circ} \mathrm{C}\right)$ with $12 \mathrm{~h}$ light/dark cycles. Standard rodent chow and water were provided ad libitum. This experimental design was approved by the committee on animal experimentation of Hiroshima International University.

\section{Joint immobilization and remobilization}

The right knees of rats in the IM, RM, and $\mathrm{RM}+\mathrm{M}$ groups were immobilized with an external fixator according to the method described in previous studies (Nagai et al. 2014, Ozawa et al. 2016). In brief, after anesthesia by intraperitoneal injection of sodium pentobarbital $(32.4 \mathrm{mg} / \mathrm{kg}$ of body weight), Kirschner wires (01-132-50; MIZUHO, Tokyo, Japan) were screwed into the femur and the tibia and were fixed by wire and resin (Provinice Fast; Matsukaze, Kyoto, Japan) to immobilize knee joints at a flexion of approximately $140^{\circ}$ (Fig. 1). During immobilization, rats could move freely using their four limbs. Knees in the control group were untreated. After three weeks, the fixation device was removed and rats in the RM and $\mathrm{RM}+\mathrm{M}$ groups were allowed to recover for one week (remobilization). It is known that one week of remobilization following three weeks of immobilization induces fibrotic reactions in the joint capsule and arthrogenic contracture progression (Kaneguchi et al. 2017, Kaneguchi et al. 2018a, Kaneguchi et al. 2018b, Kaneguchi et al. 2019). Rats in the IM group were analyzed immediately after removal of the fixator and represented data from immobilization without remobilization. Therefore, data in the control and IM groups were collected at three weeks after starting the experiment, while data in the $\mathrm{RM}$ and $\mathrm{RM}+\mathrm{M}$ groups were collected at four weeks.

\section{MMC treatment}

To inhibit remobilization-induced fibroblast proliferation, rats in the $\mathrm{RM}+\mathrm{M}$ group received intraarticular injections of $0.08 \mathrm{mg}$ of MMC (concentration $0.8 \mathrm{mg} / \mathrm{ml}$ ). Because cell proliferation is active until three days after fixator removal (Kaneguchi et al. 2017), injections were given immediately after and three days after removal. The quantity of MMC administered was determined based on the literature to achieve an antiproliferative effect (Kocaoglu et al. 2011). In the $\mathrm{RM}$ group, rats received intra-articular injections of the same volume of saline $(0.1 \mathrm{ml})$ at the same time. Thus, rats in the $\mathrm{RM}$ and $\mathrm{RM}+\mathrm{M}$ groups received two injections.

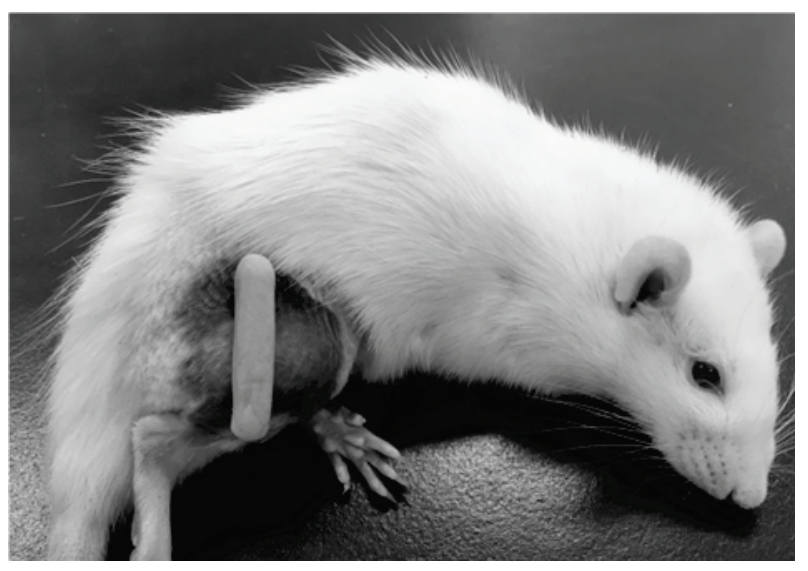

Fig. 1. Image of joint immobilization. The right knee joint is immobilized at a flexion of approximately $140^{\circ}$ (angle between the femur and the fibula is $40^{\circ}$ ) by an external fixator.

\section{ROM measurements}

At the end of experimental periods, we measured ROMs according to the method of our previous study (Kaneguchi et al. 2015). In brief, rats were anesthetized with intraperitoneal injection of sodium pentobarbital ( $32.4 \mathrm{mg} / \mathrm{kg}$ of body weight). After rat hindlimbs were skinned, rats were placed in the neutral spine position and the femur was manually fixed at $90^{\circ}$ of the hip flexion, followed by $14.6 \mathrm{~N} \mathrm{~mm}$ of knee extension moment. This moment stretched the knee joint close to its physiological limit (Moriyama et al. 2006), but did not disrupt the knee soft tissues (Trudel and Uhthoff 2000). Using a 3D motion analysis system (Kinema Tracer; Kissei Comtec, Nagano, Japan), the angle between the femur and fibula was measured as ROM before myotomy. Then, rats were sacrificed by exsanguination under sodium pentobarbital anesthesia, and knee flexor muscles were completely transected to remove the myogenic factor. Finally, ROM after myotomy was measured to evaluate arthrogenic contracture.

\section{Histological assessment}

\section{Tissue preparation}

After ROM measurements, the knee joints were harvested and immersion-fixed at flexion $90^{\circ}$ in $0.1 \mathrm{M}$ phosphate-buffered $4 \%$ paraformaldehyde ( $\mathrm{pH}$ 7.4) for $48 \mathrm{~h}$ at $4{ }^{\circ} \mathrm{C}$. After fixation, samples were decalcified by $17.7 \%$ ethylenediaminetetraacetic acid $(\mathrm{pH} 7.2$, Osteosoft; 
Merck Millipore, Darmstadt, Germany) and embedded in paraffin. Using a microtome, samples were cut into $4 \mu \mathrm{m}$ sagittal sections at the medial mid condylar level.

\section{Cell counting}

Counting of cells was performed according to the methods described in previous studies (Kaneguchi et al. 2017, Kaneguchi et al. 2018a). In brief, sections were stained with hematoxylin and eosin. We photographed the superior, central, and inferior regions of the posterior joint capsule at $40 \times$ magnification and manually counted the number of cells. Cell numbers were then converted to cells per $\mathrm{mm}^{2}$ of joint capsule.

\section{Counting of fibroblasts}

To visualize fibroblasts, we performed immunohistochemistry using anti-vimentin antibodies. Deparaffinized sections were treated with $1 \%$ trypsin for $5 \mathrm{~min}$ at $37^{\circ} \mathrm{C}$ for antigen retrieval. To quench endogenous peroxidase activity, sections were incubated with methanol containing $3 \% \mathrm{H}_{2} \mathrm{O}_{2}$ for $30 \mathrm{~min}$. Nonspecific binding was blocked by $1 \%$ normal horse serum in $0.01 \mathrm{M}$ phosphate-buffered saline (PBS; pH 7.4) for $30 \mathrm{~min}$. We then incubated sections with antivimentin antibody (1:1000 dilution; ab92547, Abcam, Cambridge, UK) for three hours at room temperature followed by rinsing with PBS. Secondary antibody (horse biotinylated anti-mouse/rabbit IgG, 1:250 dilution; BA-1400, Vector Laboratories, Burlingame, CA, USA) was added for $30 \mathrm{~min}$. After rinsing with PBS, we incubated sections with a streptavidin-biotin complex (1:50 dilution; Elite ABC, Vector Laboratories) for 30 min. Finally, immunoreactivity was visualized with a Dako EnVision + kit/HRP (DAB) (Dako Japan, Tokyo, Japan), followed by counterstaining with hematoxylin. While vimentin is often used as a fibroblast marker (Abdul et al. 2015, Glazebrook et al. 2008, Krejci et al. 2015, Wang et al. 2014), it is also expressed in other cell types, including endothelial cells, macrophages, neutrophils, and lymphocytes (Evans 1998). Therefore, spindle-shaped vimentin-positive cells not detected in luminal structures were considered fibroblasts (Kaneguchi et al. 2018a). Counting of fibroblasts was performed in the same way as cell counting.

\section{Calculating joint capsule collagen density}

We calculated collagen density following methods described previously (Kaneguchi et al. 2017). In brief, sections were stained with aldehyde fuchsin-
Masson Goldner (AFMG) to identify collagen. We captured the posterior joint capsule just behind the meniscus at 20× magnification (Fig. 2A, B) and analyzed the digitized image using ImageJ software (National Institutes of Health, Bethesda, MD, USA). To isolate collagen, we extracted green color from the original color image using the Split Channels function. Using the Threshold function, an arbitrary threshold was set to determine the green stained area. Collagen density was calculated by dividing this area by the total joint capsule area.

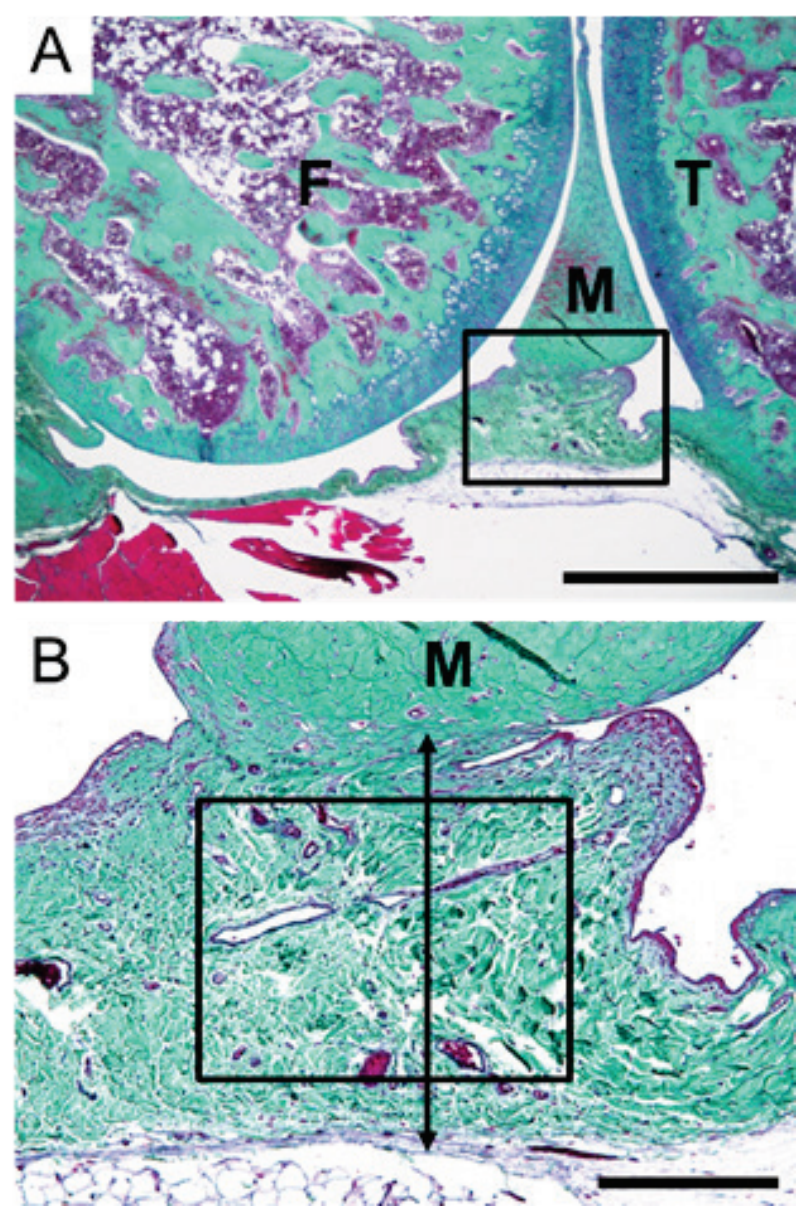

Fig. 2. Images of morphometrical and histological analyses of the posterior joint capsule. (A) shows example image of the posterior knee joint stained with aldehyde fuchsin-Masson Goldner. High magnification of the box in A is shown in B. The distance between the posterior borders of the meniscus and the joint capsule (up-down arrow) was measured as posterior joint capsule thickness (B). In addition, collagen density (percentage of green stained area) was also measured in the posterior joint capsule (box in B). F, femur; T, tibia; $M$, meniscus. Scale bars $=1 \mathrm{~mm}$ in $\mathrm{A}, 200 \mu \mathrm{m}$ in $\mathrm{B}$.

\section{Measurement of joint capsule thickness}

Using AFMG-stained sections, the thickness of the posterior joint capsule was measured according to the 
methods described previously (Kaneguchi et al. 2017). In brief, after the posterior region of the knee joint was photographed at $2 \times$ magnification, the distance between the posterior borders of the meniscus and the joint capsule was measured as joint capsule thickness using ImageJ software (Fig. 2A, B). All analyses were performed in an unblinded manner.

\section{Statistical analysis}

We performed statistical analyses using Dr. SPSS II for Windows (SPSS Japan Inc., Tokyo, Japan) and G*Power 3.1 (University of Düsseldorf, Düsseldorf, Germany). Normality of the distribution and homoge-neity of variance were tested using the Kolmogorov-Smirnov and Levene tests, respectively. All data met normality and homoscedasticity assumptions. Therefore, one-way analysis of variance (ANOVA) and the Tukey's post hoc test were applied. For all tests, a P-value of $<0.05$ was considered statistically significant. A post hoc power analysis was performed using $\mathrm{G}^{*}$ Power 3.1 .

\section{Results}

$R O M$

Knee extension ROMs before myotomy were $149 \pm 4^{\circ}, 91 \pm 4^{\circ}, 108 \pm 4^{\circ}$, and $107 \pm 5^{\circ}$ in the control, IM, $\mathrm{RM}$, and $\mathrm{RM}+\mathrm{M}$ groups, respectively (Fig. 3A). Compared with the control group, ROMs were significantly reduced in all joint-immobilized groups $(\mathrm{P}<0.001)$. Among immobilized groups, $\mathrm{ROMs}$ in the $\mathrm{RM}$ and $\mathrm{RM}+\mathrm{M}$ groups were significantly larger than that in the IM group $(\mathrm{P}<0.001)$. Between the $\mathrm{RM}$ and $\mathrm{RM}+\mathrm{M}$ groups, there was no difference in ROM before myotomy $(\mathrm{P}=0.952)$. The statistical power for ROM before myotomy was 1.00 .

Knee extension ROMs after myotomy, which can indicate restriction of ROM caused by arthrogenic factor, were $161 \pm 4^{\circ}, 139 \pm 8^{\circ}, 128 \pm 8^{\circ}$, and $138 \pm 5^{\circ}$ in the control, IM, RM, and $\mathrm{RM}+\mathrm{M}$ groups, respectively (Fig. 3B). In all joint-immobilized groups, ROMs were significantly reduced compared with the control group $(\mathrm{P}<0.001)$. Among immobilized groups, $\mathrm{ROM}$ in the RM group was significantly lower than in the IM group $(\mathrm{P}=0.019)$. However, $\mathrm{ROM}$ in the $\mathrm{RM}+\mathrm{M}$ group did not differ from the IM group ( $\mathrm{P}=0.977)$, but was significantly larger than in the $\mathrm{RM}$ group $(\mathrm{P}=0.048)$. The statistical power for ROM after myotomy was 1.00 .
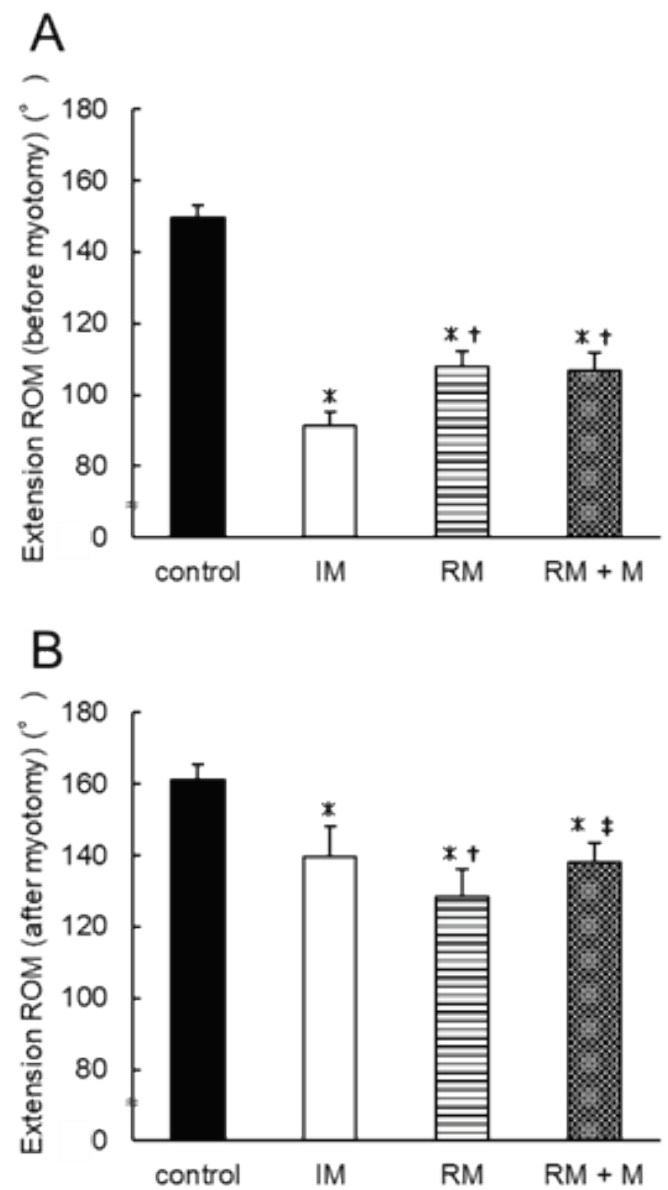

Fig. 3. Changes in knee extension ROM before and after myotomy. (A) ROM before myotomy. In the IM group, ROM before myotomy was significantly smaller than that in the control group. In the RM and RM+M groups, ROM before myotomy partially recovered, but was still significantly smaller than that in the control group. There was no difference in ROM before myotomy between the RM and RM+M groups. (B) ROM after myotomy. In the IM group, ROM after myotomy was also significantly smaller than that in the control group. In the RM group, ROM after myotomy further decreased compared with the IM group. In the RM+M group, we prevented remobilizationinduced progression of ROM restriction. Values are mean + standard deviation. * indicates significant difference compared with the control group $(\mathrm{P}<0.05) .{ }^{\dagger}$ indicates significant difference compared with the IM group $(P<0.05) .{ }^{*}$ indicates significant difference compared with the RM group $(P<0.05)$.

\section{Cellularity}

In the posterior joint capsule of the control (Fig. 4A) and IM (Fig. 4B) groups, we mainly observed spindle-shaped fibroblast-like cells and vascular endothelial cells, concluding there was no difference in cell number between these two groups $(\mathrm{P}=0.998$, $3,985 \pm 867$ and $4,084 \pm 569$ cells $/ \mathrm{mm}^{2}$, respectively, Fig. 4E). Compared with the control and IM groups, we observed a greater cell number mainly due to proliferated spindle-shaped fibroblast-like cells in the RM group 

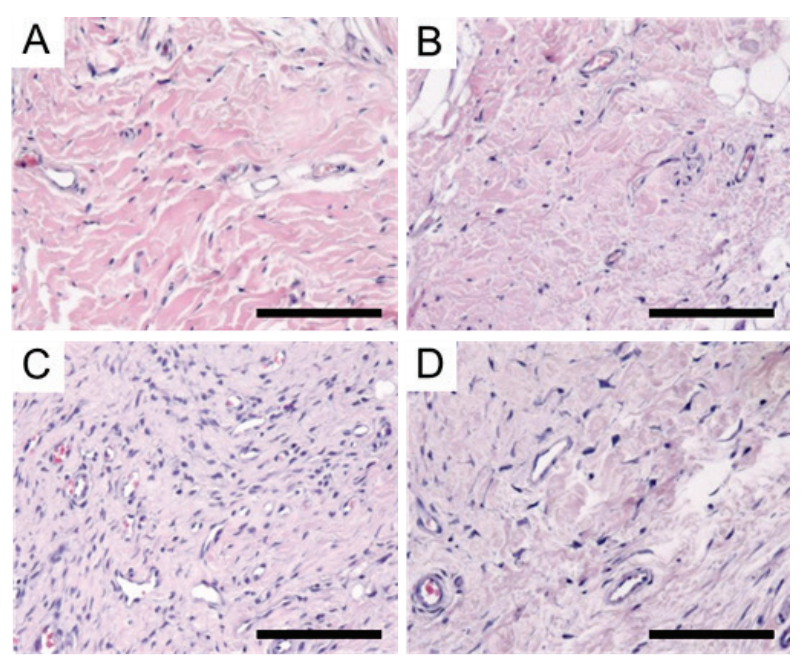

E

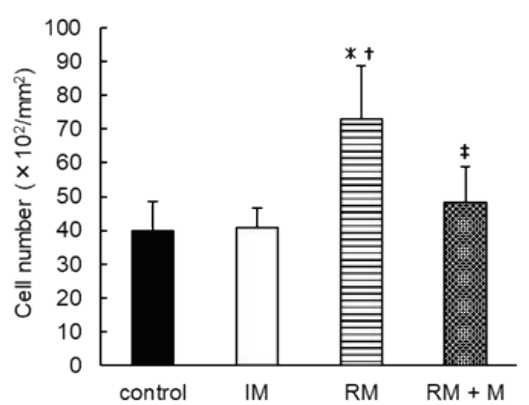

Fig. 4. Cellularity of posterior joint capsule. A-D shows the posterior knee joint capsule stained with hematoxylin and eosin. (A) control, (B) IM, (C) RM, and (D) RM+M groups. Scale bars $=100 \mu \mathrm{m}$. E shows cell number. There was no difference in cell number between the control and IM groups. In the RM group, we observed many spindle-shaped fibroblast-like cells and, consequently, cell number was significantly higher than in the control and IM groups. Remobilization-induced hypercellularity was prevented in the $\mathrm{RM}+\mathrm{M}$ group. Values are mean + standard deviation. * indicates significant difference compared with the control group $(\mathrm{P}<0.05) .{ }^{\dagger}$ indicates significant difference compared with the IM group $(P<0.05) .{ }^{\ddagger}$ indicates significant difference compared with the RM group $(\mathrm{P}<0.05)$.

( $\mathrm{P}<0.001,7,291 \pm 1,572$ cells $/ \mathrm{mm}^{2}, 183 \%$ of the control group, Fig. 4C). In the RM+M group (Fig. 4D), cell number was significantly smaller than in the RM group ( $\mathrm{P}=0.001,4,833 \pm 1,044$ cells $/ \mathrm{mm}^{2}, 66 \%$ of $\mathrm{RM}$ group $)$, but not significantly different from the control and IM groups ( $\mathrm{P}=0.468$ and 0.570 , respectively, $121 \%$ of the control group). The statistical power for cell number was 0.95 .

The number of vimentin-positive fibroblasts also did not differ between the control (Fig. 5A) and IM (Fig. 5B) groups $(\mathrm{P}=1.000, \quad 2,199 \pm 491$ and $2,180 \pm$ 393 cells $/ \mathrm{mm}^{2}$, respectively, Fig. 5E). Vimentin-positive fibroblast number in the RM group (Fig. 5C) was significantly higher than those in the control and IM groups ( $\mathrm{P}<0.001,4,434 \pm 660$ cells $/ \mathrm{mm}^{2}, 202 \%$ of the
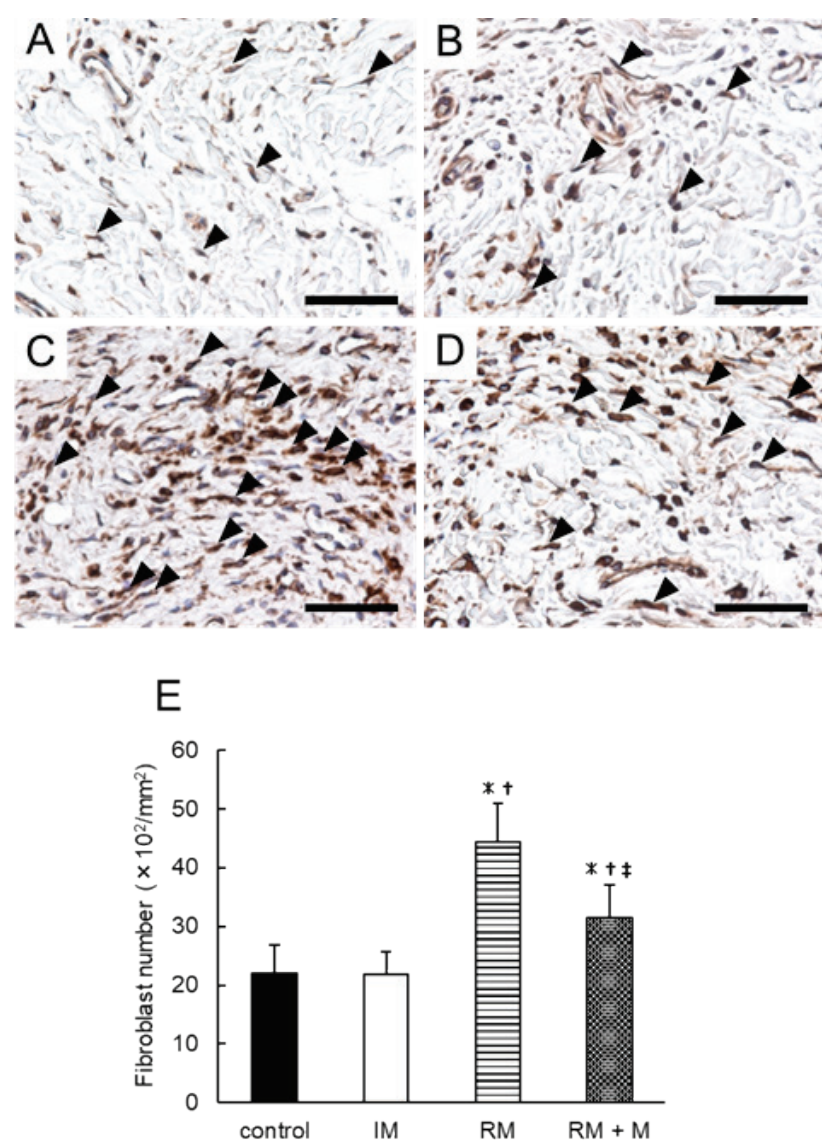

Fig. 5. Fibroblast number in the posterior joint capsule. A-D shows posterior knee joint capsule immunohistochemically stained with anti-vimentin antibody. (A) control, (B) IM, (C) RM, and (D) $R M+M$ groups. Arrowheads indicate fibroblasts. Scale bars $=50 \mu \mathrm{m}$. (E) fibroblast number. There was no difference in fibroblast number between the control and IM groups. Compared with those in the control and IM groups, the number of fibroblasts in the RM group increased. In the RM+M group, an increase in fibroblasts was partially attenuated. Values are mean + standard deviation. * indicates significant difference compared with the control group $(\mathrm{P}<0.05) .{ }^{\dagger}$ indicates significant difference compared with the IM group $(P<0.05) .{ }^{*}$ indicates significant difference compared with the RM group $(P<0.05)$.

control group). Also, in the RM+M group (Fig. 5D), vimentin-positive fibroblast number was significantly higher than those in the control and IM groups $(\mathrm{P}=0.014$ and 0.012 , respectively, $3,149 \pm 556$ cells $/ \mathrm{mm}^{2}, 143 \%$ of the control group). However, the number of vimentinpositive fibroblasts was significantly smaller in the $\mathrm{RM}+\mathrm{M}$ group than in the $\mathrm{RM}$ group $(\mathrm{P}=0.001,71 \%$ of RM group). The statistical power for fibroblast number was 1.00

\section{Collagen density}

In the control (Fig. 6A) and IM (Fig. 6B) groups, collagen fiber bundles in the posterior joint capsule were arranged with gaps. Collagen density was $54 \pm 4 \%$ and $48 \pm 8 \%$, respectively. There was no significant difference 

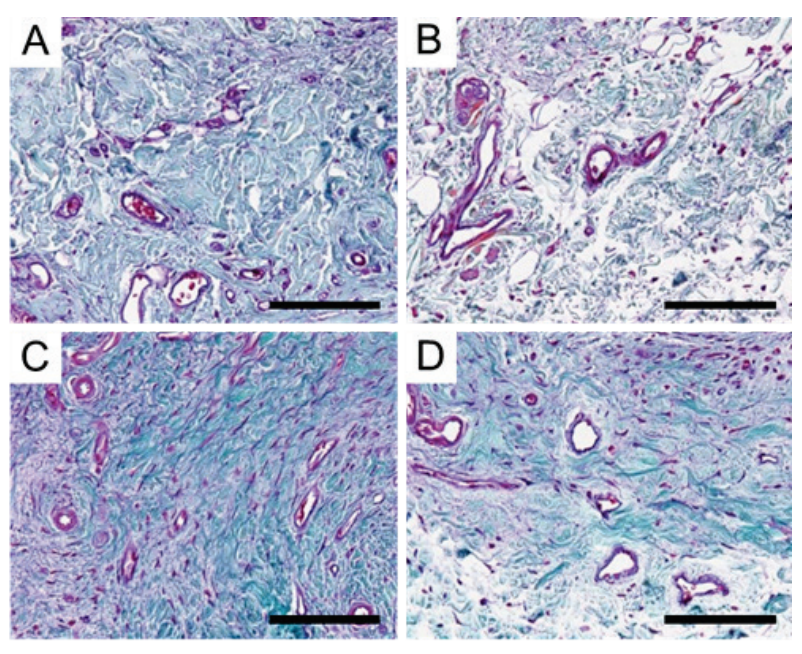

E

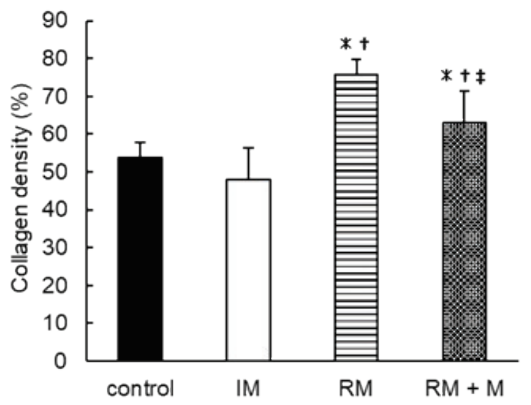

Fig. 6. Morphological changes in the posterior knee joint capsule. $A-D$ shows posterior knee joint capsule stained with aldehyde fuchsin-Masson Goldner. (A) control, (B) IM, (C) RM, and (D) $\mathrm{RM}+\mathrm{M}$ groups. Collagen is stained green. Scale bars $=100 \mu \mathrm{m}$. (E) collagen density. There was no difference in collagen density between the control and IM groups. In the RM group, the gaps of collagen bundles narrowed and joint capsules became denser. Consequently, collagen density in the RM group significantly increased compared with the control and IM groups. In the $R M+M$ group, a remobilization-induced increase in collagen density was partially attenuated. Values are mean + standard deviation. * indicates significant difference compared with the control group $(P<0.05) .{ }^{+}$indicates significant difference compared with the IM group $(P<0.05) .{ }^{*}$ indicates significant difference compared with the RM group $(P<0.05)$.

in collagen density between the control and IM groups ( $\mathrm{P}=0.324$, Fig. 6E). In the RM group (Fig. 6C), the gap of collagen fiber bundles disappeared and collagen density increased to $76 \pm 4 \%$, significantly different to the control and IM groups $(\mathrm{P}<0.001)$. In the $\mathrm{RM}+\mathrm{M}$ group (Fig. $6 \mathrm{D}$ ), gaps between collagen fiber bundles were partially restored and collagen density was $63 \pm 8 \%$. Collagen density in the $\mathrm{RM}+\mathrm{M}$ group was significantly lower than that in the RM group $(\mathrm{P}=0.006)$, but significantly higher than in the control and IM groups $(\mathrm{P}=0.043$ and 0.001 , respectively). The statistical power for collagen density was 1.00 .
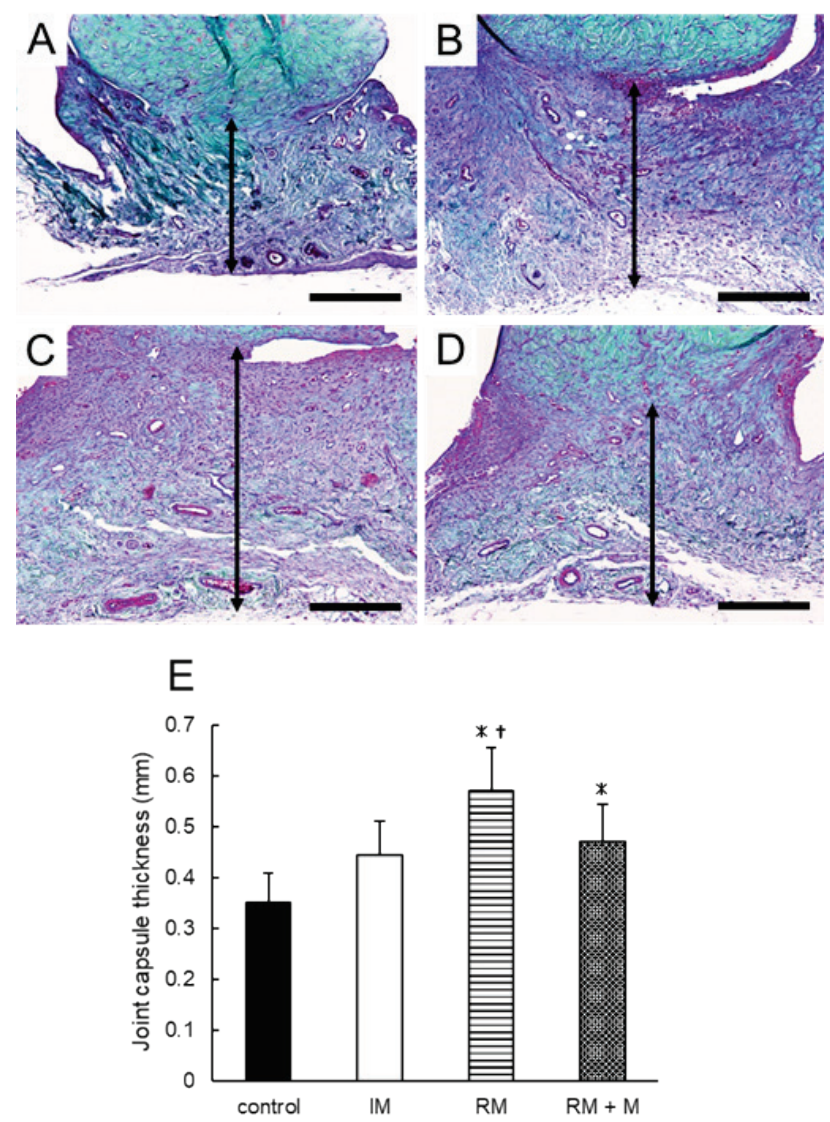

Fig. 7. Thickness in the posterior joint capsule. A-D shows posterior knee joint capsule stained with aldehyde fuchsinMasson Goldner. (A) control, (B) IM, (C) RM, and (D) $R M+M$ groups. Up-down arrows indicate posterior joint capsule thickness. Scale bars $=200 \mu \mathrm{m}$. (E) joint capsule thickness. Compared with the control group, the posterior joint capsule in the IM group was slightly thickened, but differences were not significant $(P=0.077)$. Joint capsule thickness in the RM group was significantly larger than those in the control and IM groups. In the RM+M group, joint capsule thickness tended to be smaller than in the RM groups $(P=0.067)$ and was comparable to the IM group. Values are mean + standard deviation. * indicates significant difference compared with the control group $(P<0.05) .{ }^{+}$indicates significant difference compared with the IM group $(P<0.05)$.

\section{Joint capsule thickness}

In the control group (Fig. 7A), joint capsule thickness was $0.35 \pm 0.06 \mathrm{~mm}$ (Fig. 7E). In the IM group (Fig. 7B), the posterior joint capsule was slightly thickened $(0.45 \pm 0.07 \mathrm{~mm})$, but not significantly, compared with that in the control group $(\mathrm{P}=0.077)$. Joint capsule thickness in the RM group was $0.57 \pm 0.09 \mathrm{~mm}$ (Fig. 7C) and was significantly thicker than those in the control and IM groups $(\mathrm{P}<0.001$ and $\mathrm{P}=0.014$, respectively). In the $\mathrm{RM}+\mathrm{M}$ group (Fig. 7D), joint capsule thickness tended to be lower than in the RM groups $\quad(0.47 \pm 0.07 \mathrm{~mm}, \quad \mathrm{P}=0.067) \quad$ and was 
comparable to the IM group $(\mathrm{P}=0.901)$. The statistical power for joint capsule thickness was 0.98 .

\section{Discussion}

In this study, we tested whether MMC administration prevents the progression of remobilization-induced arthrogenic contracture by inhibiting fibroblast proliferation and fibrosis in the joint capsule. As expected, MMC injections served to attenuate progression of arthrogenic contracture by suppressing fibroblast proliferation, leading to significantly lower collagen density and a tendency to be lower joint capsule thickness. These results suggest that joint capsule fibrosis is a potential cause of arthrogenic contracture progression in remobilized joints.

Three weeks of immobilization significantly reduced ROMs both before and after myotomy. After one week of remobilization, ROM before myotomy increased but after myotomy further decreased. These results indicate that remobilization improves myogenic contracture, but aggravates arthrogenic contracture oppositely. Although immobilization-induced arthrogenic contracture is not improved by remobilization (Kaneguchi et al. 2017, Kaneguchi et al. 2018a, Kaneguchi et al. 2018b, Kaneguchi et al. 2019, Trudel et al. 2014), arthrogenic contracture in intra-articular adhesion model using joint immobilization with intraarticular injury partially recovers after remobilization (Baranowski et al. 2018). These findings suggest that arthrogenic contracture attributed to intra-articular injury may be variable in contrast with immobilization only. Intra-articular injections of MMC during remobilization could prevent further decrease in ROM after myotomy, which may be due to intra-articular (micro) injury (Kaneguchi et al. 2017). However, MMC injections did not improve ROM before myotomy. Our results indicate that MMC has beneficial effects on arthrogenic contracture but not on myogenic one.

Fibrosis in joint components strongly contributes to progressing arthrogenic contracture in remobilized (Kaneguchi et al. 2017, Kaneguchi et al. 2018a, Kaneguchi et al. 2018b) as well as injured joints (Fukui et al. 2000, Fukui et al. 2001, Gao et al. 2017, Li et al. 2013a). Further, we observed increased joint capsule thickness and collagen density in the joint capsule following remobilization together with progressing arthrogenic contracture. Extracellular matrix proteins, such as collagen, are produced by fibroblasts. In joint injury-induced contracture, inhibiting fibroblast proliferation by anti-proliferative agents MMC or hydroxycamptothecin can attenuate fibrosis in intraarticular adhesion sites (Li et al. 2013b, Liang et al. 2014). Therefore, it seems likely that fibroblast proliferation plays an important role in generating joint fibrosis. In our present and previous studies, we observed development of joint capsule fibrosis together with fibroblast proliferation during remobilization (Kaneguchi et al. 2017, Kaneguchi et al. 2018a, Kaneguchi et al. 2018b, Kaneguchi et al. 2019), suggesting that fibroblast proliferation contributes to fibrosis formation in remobilized joints. We previously revealed that administering the steroidal anti-inflammatory drug dexamethasone during remobilization can prevent joint capsule fibrosis by suppressing hypercellularity with increasing fibroblast numbers (Kaneguchi et al. 2018b). In the present study, administering MMC, which selectively prevents cell proliferation without inhibiting inflammation, was also able to attenuate joint capsule fibrosis; this prevented arthrogenic contracture progression during remobilization. We previously reported that inflammatory reactions reach their peak within one day of remobilization, but that arthrogenic contracture progression is not observed at that point (Kaneguchi et al. 2017). Progression of arthrogenic contracture characterized by development of joint capsule fibrosis is observed only after inflammation (Kaneguchi et al. 2017). These findings suggest that fibroblast proliferation (and subsequent upregulation of collagens) triggered by inflammation plays an important role in driving fibrotic processes, which induces arthrogenic contracture progression in remobilized joints.

In immobilized joints, remobilization can improve myogenic (Kaneguchi et al. 2017, Trudel et al. 2014) but not arthrogenic contracture (Kaneguchi and Ozawa 2017, Kaneguchi et al. 2017, Kaneguchi et al. 2018a, Kaneguchi et al. 2018b, Kaneguchi et al. 2019, Trudel et al. 2014). Therefore, effective therapeutic interventions for arthrogenic contracture are needed to avoid permanent joint contracture. Some animal and clinical studies reported the effectiveness of antiinflammatory and anti-fibrotic agents on the attenuation of joint contracture (Baranowski et al. 2019, Usher et al. 2019). In the clinical setting, however, treatment options for joint contracture are limited to passive joint movements such as stretching, manipulation under anaesthesia (MUA), or surgical treatments (Charalambous and Morrey 2012, Wong et al. 2015). 
Previous studies identify no positive effects of stretching on joint contracture (Harvey et al. 2017a, Harvey et al. 2017b, Moseley et al. 2005). MUA and surgical treatments are effective to improve joint contracture, but have the risk of complications, including nerve symptoms, heterotopic ossification, and instability (Cai et al. 2015, Usher et al. 2019). Developing new therapeutic strategies as alternatives to current treatments is therefore a critical concern (Wong et al. 2015). In this study, we demonstrated that intra-articular injections of the cell cycle inhibitor MMC effectively attenuates remobilization-induced joint capsule fibrosis and arthrogenic contracture progression. Inhibiting fibroblast proliferation during remobilization may become a novel therapeutic strategy in treating immobilization-induced arthrogenic contracture.

This study has some limitations. First, all analyses were performed in an unblinded manner. We thus cannot exclude the possibility of the subjective bias. Second, we immobilized knee joint using external fixator constructed by Kirschner wires, wire, and resin. Cast immobilization might be more suitable to mimic the human situation after the orthopedic disorders. Third, immunohistochemistry was performed only for the fibroblast. Both fibroblasts and myofibroblasts are major contributors to fibrotic changes (Hinz et al. 2012, Kendall and Feghali-Bostwick 2014). In particular, myofibroblasts expressing $\alpha$-smooth muscle actin ( $\alpha$-SMA) have high extracellular matrix protein production capacity (Hinz et al. 2012, Kendall and Feghali-Bostwick 2014) and thus play a central role in joint fibrosis (Baranowski et al. 2019, Li et al. 2013b). Identification of myofibroblasts by $\alpha$-SMA would give valuable information.

In conclusion, the present study demonstrated that inhibiting fibroblast proliferation by intra-articular MMC injections during remobilization can benefit the treatment of arthrogenic contracture by attenuating joint capsule fibrosis.

\section{Conflict of Interest}

There is no conflict of interest.

\section{Acknowledgements}

This study was supported by a grant from the Japanese Physical Therapy Association.

\section{References}

ABDUL N, DIXON D, WALKER A, HORABIN J, SMITH N, WEIR DJ, BREWSTER NT, DEEHAN DJ, MANN DA, BORTHWICK LA: Fibrosis is a common outcome following total knee arthroplasty. Sci Rep 5: 16469, 2015. https://doi.org/10.1038/srep16469

BARANOWSKI A, SCHLEMMER L, FORSTER K, MATTYASOVSZKY SG, RITZ U, WAGNER D, ROMMENS PM, HOFMANN A: A novel rat model of stable posttraumatic joint stiffness of the knee. J Orthop Surg Res 13: 185, 2018. https://doi.org/10.1186/s13018-018-0894-y

BARANOWSKI A, SCHLEMMER L, FORSTER K, SLOTINA E, MICKAN T, TRUFFEL S, KLEIN A, MATTYASOVSZKY SG, HOFMANN A, RITZ U, ROMMENS PM: Effects of losartan and atorvastatin on the development of early posttraumatic joint stiffness in a rat model. Drug Des Devel Ther 13: 2603-2618, 2019. https://doi.org/10.2147/DDDT.S204135

BOT AG, SOUER JS, VAN DIJK CN, RING D: Association between individual DASH tasks and restricted wrist flexion and extension after volar plate fixation of a fracture of the distal radius. Hand (N Y) 7: 407-412, 2012. https://doi.org/10.1007/s11552-012-9447-8

CAI J, WANG W, YAN H, SUN Y, CHEN W, CHEN S, FAN C: Complications of open elbow arthrolysis in post-traumatic elbow stiffness: a systematic review. PLoS One 10: e0138547, 2015. https://doi.org/10.1371/journal.pone.0138547

CHARALAMBOUS CP, MORREY BF: Posttraumatic elbow stiffness. J Bone Joint Surg Am 94: 1428-1437, 2012. https://doi.org/10.2106/JBJS.K.00711

CHESWORTH BM, VANDERVOORT AA: Comparison of passive stiffness variables and range of motion in uninvolved and involved ankle joints of patients following ankle fractures. Phys Ther 75: 253-261, 1995. https://doi.org/10.1093/ptj/75.4.253

DE SMET L: Does restricted wrist motion influence the disability of the upper limb? Acta Orthop Belg 73: 446-450, 2007. 
EMAMI MJ, JABERI FM, AZARPIRA N, VOSOUGHI AR, TANIDEH N: Prevention of arthrofibrosis by monoclonal antibody against vascular endothelial growth factor: a novel use of bevacizumab in rabbits. Orthop Traumatol Surg Res 98: 759-764, 2012. https://doi.org/10.1016/j.otsr.2012.05.020

EVANS RM: Vimentin: the conundrum of the intermediate filament gene family. Bioessays 20: 79-86, 1998. https://doi.org/10.1002/(SICI)1521-1878(199801)20:1<79::AID-BIES11>3.0.CO;2-5

FUKUI N, TASHIRO T, HIRAOKA H, ODA H, NAKAMURA K: Adhesion formation can be reduced by the suppression of transforming growth factor-beta1 activity. J Orthop Res 18: 212-219, 2000. https://doi.org/10.1002/jor.1100180208

FUKUI N, FUKUDA A, KOJIMA K, NAKAJIMA K, ODA H, NAKAMURA K: Suppression of fibrous adhesion by proteoglycan decorin. J Orthop Res 19: 456-462, 2001. https://doi.org/10.1016/S0736-0266(00)90016-0

GAO ZY, WU JX, LIU WB, SUN JK: Reduction of adhesion formation after knee surgery in a rat model by botulinum toxin A. Biosci Rep 37, 2017. https://doi.org/10.1042/BSR20160460

GLAZEBROOK MA, WRIGHT JR JR, LANGMAN M, STANISH WD, LEE JM: Histological analysis of Achilles tendons in an overuse rat model. J Orthop Res 26: 840-846, 2008. https://doi.org/10.1002/jor.20546

HAGIWARA Y, ANDO A, ONODA Y, MATSUI H, CHIMOTO E, SUDA H, ITOI E: Expression patterns of collagen types I and III in the capsule of a rat knee contracture model. J Orthop Res 28: 315-321, 2010. https://doi.org/10.1002/jor.20997

HARVEY LA, KATALINIC OM, HERBERT RD, MOSELEY AM, LANNIN NA, SCHURR K: Stretch for the treatment and prevention of contractures. Cochrane Database Syst Rev 1: CD007455, $2017 \mathrm{a}$. https://doi.org/10.1002/14651858.CD007455.pub3

HARVEY LA, KATALINIC OM, HERBERT RD, MOSELEY AM, LANNIN NA, SCHURR K: Stretch for the treatment and prevention of contracture: an abridged republication of a Cochrane Systematic Review. J Physiother 63: 67-75, 2017b. https://doi.org/10.1016/j.jphys.2017.02.014

HINZ B, PHAN SH, THANNICKAL VJ, PRUNOTTO M, DESMOULIERE A, VARGA J, DE WEVER O, MAREEL M, GABBIANI G: Recent developments in myofibroblast biology: paradigms for connective tissue remodeling. Am J Pathol 180: 1340-1355, 2012. https://doi.org/10.1016/j.ajpath.2012.02.004

KAHMANN L, BEYER U, MEHLHORN G, THIEL FC, STRNAD V, FASCHING PA, LUX MP: Mitomycin C in patients with gynecological malignancies. Onkologie 33: 547-557, 2010. https://doi.org/10.1159/000319742

KANEGUCHI A, OZAWA J, MORIYAMA H, YAMAOKA K: Structures responsible for the formation of knee joint contracture secondary to adjuvant-induced arthritis in a rat model. Iryo Kogaku Zassi (J Med Eng): 1-12, 2015.

KANEGUCHI A, OZAWA J: The preventive effects of low-level laser therapy on arthrogenic contracture progression in remobilized rat knee. (Article in Japanese) Jpn J Electrophys Agents 24: 47-51, 2017.

KANEGUCHI A, OZAWA J, KAWAMATA S, YAMAOKA K: Development of arthrogenic joint contracture as a result of pathological changes in remobilized rat knees. J Orthop Res 35: 1414-1423, 2017. https://doi.org/10.1002/jor.23419

KANEGUCHI A, OZAWA J, MINAMIMOTO K, YAMAOKA K: Active exercise on immobilization-induced contractured rat knees develops arthrogenic joint contracture with pathological changes. J Appl Physiol (1985) 124: 291-301, 2018a. https://doi.org/10.1152/japplphysiol.00438.2017

KANEGUCHI A, OZAWA J, YAMAOKA K: Anti-inflammatory drug dexamethasone treatment during the remobilization period improves range of motion in a rat knee model of joint contracture. Inflammation 41: 1409-1423, 2018b. https://doi.org/10.1007/s10753-018-0788-5

KANEGUCHI A, OZAWA J, MINAMIMOTO K, YAMAOKA K: Low-level laser therapy prevents treadmill exerciseinduced progression of arthrogenic joint contracture via attenuation of inflammation and fibrosis in remobilized rat knees. Inflammation 42: 857-873, 2019. https://doi.org/10.1007/s10753-018-0941-1

KENDALL RT, FEGHALI-BOSTWICK CA: Fibroblasts in fibrosis: novel roles and mediators. Front Pharmacol 5: 123, 2014. https://doi.org/10.3389/fphar.2014.00123

KOCAOGLU B, AKGUN U, NALBANTOGLU U, POYANLI O, KARAHAN M: Adhesion reduction after knee surgery in a rat model by mitomycin C. Knee Surg Sports Traumatol Arthrosc 19: 94-98, 2011. https://doi.org/10.1007/s00167-010-1154-9 
KONDO Y, NAKANO J, SAKAMOTO J, KATAOKA H, YOKOYAMA S, HONDA Y, ORIGUCHI T, YOSHIMURA T, OKITA M: Effects of prolonged stretching and thermotherapy on muscle contracture of immobilized rat soleus muscle. J Phys Ther Sci 24: 541-547, 2012. https://doi.org/10.1589/jpts.24.541

KREJCI E, KODET O, SZABO P, BORSKY J, SMETANA K JR, GRIM M, DVORANKOVA B: In vitro differences of neonatal and later postnatal keratinocytes and dermal fibroblasts. Physiol Res 64: 561-569, 2015.

LANE HA, SWALE JA, MAJMUDAR PA: Prophylactic use of mitomycin-C in the management of a buttonholed LASIK flap. J Cataract Refract Surg 29: 390-392, 2003. https://doi.org/10.1016/S0886-3350(02)01434-7

LI F, HE B, LIU S, FAN C: Celecoxib effectively inhibits the formation of joint adhesions. Exp Ther Med 6: 1507-1511, 2013a. https://doi.org/10.3892/etm.2013.1336

LI F, LIU S, FAN C: Lentivirus-mediated ERK2 siRNA reduces joint capsule fibrosis in a rat model of post-traumatic joint contracture. Int J Mol Sci 14: 20833-20844, 2013b. https://doi.org/10.3390/ijms141020833

LI Y, MA X, YU P, WANG S: Intra-articular adhesion reduction after knee surgery in rabbits by calcium channel blockers. Med Sci Monit 20: 2466-2471, 2014. https://doi.org/10.12659/MSM.892957

LIANG Y, SUN Y, LI X, YAN L, WANG J, HU J, YU H, XIAO H, CHEN H, SUN Z, CAI J, FENG X, XIONG C, HE J: The optimal concentration of topical hydroxycamptothecin in preventing intraarticular scar adhesion. Sci Rep 4: 4621, 2014. https://doi.org/10.1038/srep04621

MORIYAMA H, YOSHIMURA O, SUNAHORI H, TOBIMATSU Y: Comparison of muscular and articular factors in the progression of contractures after spinal cord injury in rats. Spinal Cord 44: 174-181, 2006. https://doi.org/10.1038/sj.sc.3101802

MORIYAMA H, YOSHIMURA O, KAWAMATA S, TAKEMOTO H, SAKA Y, TOBIMATSU Y: Alteration of knee joint connective tissues during contracture formation in spastic rats after an experimentally induced spinal cord injury. Connect Tissue Res 48: 180-187, 2007. https://doi.org/10.1080/03008200701413512

MOSELEY AM, HERBERT RD, NIGHTINGALE EJ, TAYLOR DA, EVANS TM, ROBERTSON GJ, GUPTA SK, PENN J: Passive stretching does not enhance outcomes in patients with plantarflexion contracture after cast immobilization for ankle fracture: a randomized controlled trial. Arch Phys Med Rehabil 86: 1118-1126, 2005. https://doi.org/10.1016/j.apmr.2004.11.017

NAGAI M, AOYAMA T, ITO A, IIJIMA H, YAMAGUCHI S, TAJINO J, ZHANG X, AKIYAMA H, KUROKI H: Contributions of biarticular myogenic components to the limitation of the range of motion after immobilization of rat knee joint. BMC Musculoskelet Disord 15: 224, 2014. https://doi.org/10.1186/1471-2474-15-224

NAVA MB, ROCCO N, CATANUTO G, FRANGOU J, RISPOLI C, OTTOLENGHI J, BRUNO N, SPANO A: Role of mitomycin $\mathrm{C}$ in preventing capsular contracture in implant-based reconstructive breast surgery: randomized controlled trial. Plast Reconstr Surg 139: 819-826, 2017. https://doi.org/10.1097/PRS.0000000000003170

NIGHTINGALE EJ, MOSELEY AM, HERBERT RD: Passive dorsiflexion flexibility after cast immobilization for ankle fracture. Clin Orthop Relat Res 456: 65-69, 2007. https://doi.org/10.1097/BLO.0b013e31802fc161

OKITA M, YOSHIMURA T, NAKANO J, SAEKI A, UEHARA A, MINESHITA A, EGUCHI K: Effects of short duration stretching on disuse muscle atrophy in immobilized rat soleus muscles. J Jpn Phys Ther Assoc 4: 1-5, 2001. https://doi.org/10.1298/jijpta.4.1

OZAWA J, KANEGUCHI A, TANAKA R, KITO N, MORIYAMA H: Cyclooxygenase-2 inhibitor celecoxib attenuates joint contracture following immobilization in rat knees. BMC Musculoskelet Disord 17: 446, 2016. https://doi.org/10.1186/s12891-016-1303-5

SASABE R, SAKAMOTO J, GOTO K, HONDA Y, KATAOKA H, NAKANO J, ORIGUCHI T, ENDO D, KOJI T, OKITA M: Effects of joint immobilization on changes in myofibroblasts and collagen in the rat knee contracture model. J Orthop Res 35: 1998-2006, 2017. https://doi.org/10.1002/jor.23498

SIDLE DM, KIM H: Keloids: prevention and management. Facial Plast Surg Clin North Am 19: 505-515, 2011. https://doi.org/10.1016/j.fsc.2011.06.005

SIMMAN R, ALANI H, WILLIAMS F: Effect of mitomycin C on keloid fibroblasts: an in vitro study. Ann Plast Surg 50: 71-76, 2003. https://doi.org/10.1097/00000637-200301000-00012

TRUDEL G, UHTHOFF HK: Contractures secondary to immobility: is the restriction articular or muscular? An experimental longitudinal study in the rat knee. Arch Phys Med Rehabil 81: 6-13, 2000. https://doi.org/10.1053/apmr.2000.0810006 
TRUDEL G, LANEUVILLE O, COLETTA E, GOUDREAU L, UHTHOFF HK: Quantitative and temporal differential recovery of articular and muscular limitations of knee joint contractures; results in a rat model. J Appl Physiol (1985) 117: 730-737, 2014. https://doi.org/10.1152/japplphysiol.00409.2014

USHER KM, ZHU S, MAVROPALIAS G, CARRINO JA, ZHAO J, XU J: Pathological mechanisms and therapeutic outlooks for arthrofibrosis. Bone Res 7: 9, 2019. https://doi.org/10.1038/s41413-019-0047-X

USUBA M, AKAI M, SHIRASAKI Y, MIYAKAWA S: Experimental joint contracture correction with low torque--long duration repeated stretching. Clin Orthop Relat Res 456: 70-78, 2007. https://doi.org/10.1097/BLO.0b013e31803212bf

WANG J, YAN L, SUN Y, WANG D, DAI S, YU T, GU J, JIANG B, FENG X, HU H, WANG Q, YIN B, LV G: A comparative study of the preventive effects of mitomycin $\mathrm{C}$ and chitosan on intraarticular adhesion after knee surgery in rabbits. Cell Biochem Biophys 62: 101-105, 2012. https://doi.org/10.1007/s12013-011-9266-5

WANG Z, WANG Y, XIE P, LIU W, ZHANG S: Calcium channel blockers in reduction of epidural fibrosis and dural adhesions in laminectomy rats. Eur J Orthop Surg Traumatol 24 (Suppl 1): S293-S298, 2014. https://doi.org/10.1007/s00590-013-1395-7

WONG K, TRUDEL G, LANEUVILLE O: Noninflammatory joint contractures arising from immobility: animal models to future treatments. Biomed Res Int 2015: 848290, 2015. https://doi.org/10.1155/2015/848290 